\title{
A fatal multiple-lesion, inflammatory, pseudotumor in the lung: a rare case report
}

\author{
Na Li, Changpeng Zou, Siming Gao, Ying Guo, Wei Wang, Yan Guo, Juan Zhang, Cong Wang, Yonghui An \\ Department of Oncology, The First Hospital of Hebei Medical University, Shijiazhuang, China \\ Correspondence to: Yonghui An. Department of Oncology, The First Hospital of Hebei Medical University, Shijiazhuang 050000, China. \\ Email: sjzyhd@vip.sina.com.
}

\begin{abstract}
An inflammatory pseudotumor is considered a benign form of lesion marked by a proliferation of myofibroblasts with different degrees of inflammatory infiltrates. Pulmonary inflammatory pseudotumors (PIPs) are extremely rare in middle-aged adults. Normally, a PIP has a single lesion, and can be controlled constantly by surgery and drugs. In this paper, we report a case study of a 51-year-old male patient who presented with multiple inflammatory pseudotumors in lungs, thoracic spine, ribs, left humerusl, derived from PIPs throughout his body, which indicated a long disease term and significant recrudescence. After 6 surgeries (a wedge resection of the right lower lobe, a removal of three thoracic vertebral lesions, a removal of left humeral tumor lesion, a right lower lobe resection, local cryoablation of right lung, debridement of left upper-arm osteomyelitis and soft tissue infection), radiotherapy for lesions of left humerus destruction at a total dose of $20 \mathrm{~Gy} / 10 \mathrm{~F}$, and systematic treatments $(30 \mathrm{mg}$ prednisone acetate daily for 6 weeks, $50 \mathrm{mg}$ compound cyclophosphamide tablets for 2 weeks; antibiotics, blood transfusions, nutritional support), his symptoms improved but reoccurred. The patient ultimately died of septic shock. Our case report highlights that the progression of a PIP to a malignant form requires further research. A multiple-lesion PIP that does not respond to systemic treatment can be highly dangerous.
\end{abstract}

Keywords: Inflammatory pseudotumor; pulmonary pseudotumor; inflammatory myofibroblastic tumor; lung tumor; case report

Submitted Feb 09, 2021. Accepted for publication Apr 14, 2021.

doi: $10.21037 /$ tcr-21-564

View this article at: http://dx.doi.org/10.21037/tcr-21-564

\section{Introduction}

An inflammatory pseudotumor is a rare tumor. It has non-specific clinical and radiological manifestations (1). The inflammatory pseudotumor was first identified in a lung specimen in 1939, and has been classified as a soft tissue tumor since 2002 (2). Pulmonary inflammatory pseudotumors (PIPs) are extremely rare in adults. Pathologically, A PIP is marked by the proliferation of myofibroblasts with different degrees of inflammatory infiltrates, and is generally classified as a benign tumor; however, it may have a tendency to become malignant (e.g., to develop into a calcifying fibrous tumor complicated by empyema) (3). Limited reports on PIPs have suggested that such tumors normally have a single lesion, and can be controlled constantly by surgery and drugs (4).

We present a case report of a patient with multiple inflammatory pseudotumors derived from PIPs throughout the body. These PIPs indicated a long disease term and significant recrudescence. In our case report, the disease had a high mortality and was malignant. To date, no other similar cases appear to have been reported. Our report provides novel insights into this extremely rare disease, and highlights that a PIP may be more dangerous than previously thought. We present the following article in accordance with the CARE reporting checklist (available at 
http://dx.doi.org/10.21037/tcr-21-564).

\section{Case presentation}

A 51-year-old male reported that he had been experiencing right chest and back pain for more than 2 months. The pain produced an inactive condition. The patient had no family and other medical history. The man was admitted as a patient to our hospital on January $15^{\text {th }}, 2013$. Previously, a biopsy (a needle aspiration) had been performed to diagnose the right lower pulmonary pseudotumor. The patient had also received a wedge resection of the right lower lobe at Hebei Medical University, The Fourth Hospital in May 2011. A computerized tomography (CT) scan showed a mass in the lower lobe of the right lung accompanied by bone destruction of the 7,8 , and 9 thoracic vertebrae, which were suspected to have metastasized. Multiple enlarged lymph nodes were observed in the right hilar. Bilateral pleural hypertrophy was also observed, and fibre cord signs were seen in the lower lobe of both lungs. These were considered lesions after the resection of the inflammatory pseudotumor of the right lower lobe. Written informed consent was obtained from the patient for publication of this study and any accompanying images. All procedures performed in studies involving human participants were in accordance with the ethical standards of the institutional and/or national research committee(s) and with the Helsinki Declaration (as revised in 2013).

The diagnosis of the thoracic spine space was definite, and the previous conservative treatment was deemed invalid. On January 22, 2013, the patient was placed under general anesthesia, and three thoracic vertebral lesions were removed, and the right lung lesions were subject to a needle aspiration. The lesioned tissue was sent for pathological examination. The results revealed the following: (I) the acute and chronic purulent inflammations were observed, (II) the tissue showed inflammatory granulation and granuloma; and (III) tuberculosis could not be excluded. The sputum (bacterial) culture results were negative.

The following results were drawn from the postoperative pathological consultations: (I) In the lung tissues of the right lower lung and left lung, there were large amounts of neutrophils, lymphocytes, monocytes, scattered eosinophils, multinucleated giant-cell infiltration, and fibrinous exudation, and organization in the alveolar cavity; and (II) in the thoracic vertebrae sections, there were large amounts of neutrophils, lymphocytes, monocytes, scattered multinucleated giant-cell infiltration, and significant focal necrosis (within which neutrophil aggregation and abscesses were observed). These results suggested a special infectious disease with non-caseating necrosis, neutrophil aggregation, and abscesses. The pathological manifestations did not support a diagnosis of tuberculosis. Based on these results, the Department of Hematology was asked to consult on the case, and a bone biopsy was undertaken. The results revealed increased plasma cells. Myeloma could not be excluded.

Next, the patient presented with a sudden high fever of $40{ }^{\circ} \mathrm{C}$. Antibiotics and antipyretic treatment were administered, and the patient's symptoms improved. In March, the patient reported that his left upper arm was painful. On March $3^{\text {rd }}, 2013$, CT imaging revealed the following: (I) destruction in the $8^{\text {th }}$ to $10^{\text {th }}$ ribs (malignant tumors were suspected); (II) a small amount of pleural effusion on the right chest; (III) a solitary nodule in the posterior segment of the left upper lobe of the lung (malignant tumors were suspected); (IV) striped highdensity shadows in the lower lobe of the right lung (which were thought to be inflammatory lesions); and (V) multiple enlarged lymph nodes in the mediastinum. Additionally, malignant bone tumors in the left humerus were suspected.

The patient underwent left humeral tumor lesion removal on March $19^{\text {th }}, 2013$. The chiseled bone cortex and intramedullary curettage of the lesion tissue were sent for pathological examination; and some pus samples were cultured for drug sensitivity tests. The postoperative pathological results revealed the following: (I) in the left humeral shaft, osteoma-like hyperplasia existed; and (II) in the left humeral bone marrow cavity, the hyperplasia was accompanied by hemorrhaging, and inflammatory granulation tissues and granulomas had formed. A postoperative culture showed no bacterial growth in the patient's left humeral bone marrow cavity. On April $1^{\text {st }}$, a postoperative CT scan (see Figure $1 A$ ) suggested a diagnosis of peripheral lung cancer in the left upper lobe, and an inflammatory pseudotumor was also suspected. The enhanced CT plain scan showed that there was a round soft tissue density mass in the lower lobe of the right lung (enhanced signals), and the lesion was closely related to the thoracic 7 to 9 vertebrae. The pain was considered to be caused by this right lower lobe lesion.

On April $10^{\text {th }}, 2013$, a right lower lobe resection was undertaken. After the patient was placed under general anesthesia, his skin, subcutaneous and muscular layer were incised, and his thoracic cavity was exposed. However, extensive adhesions were found in the cavity, and the lower 

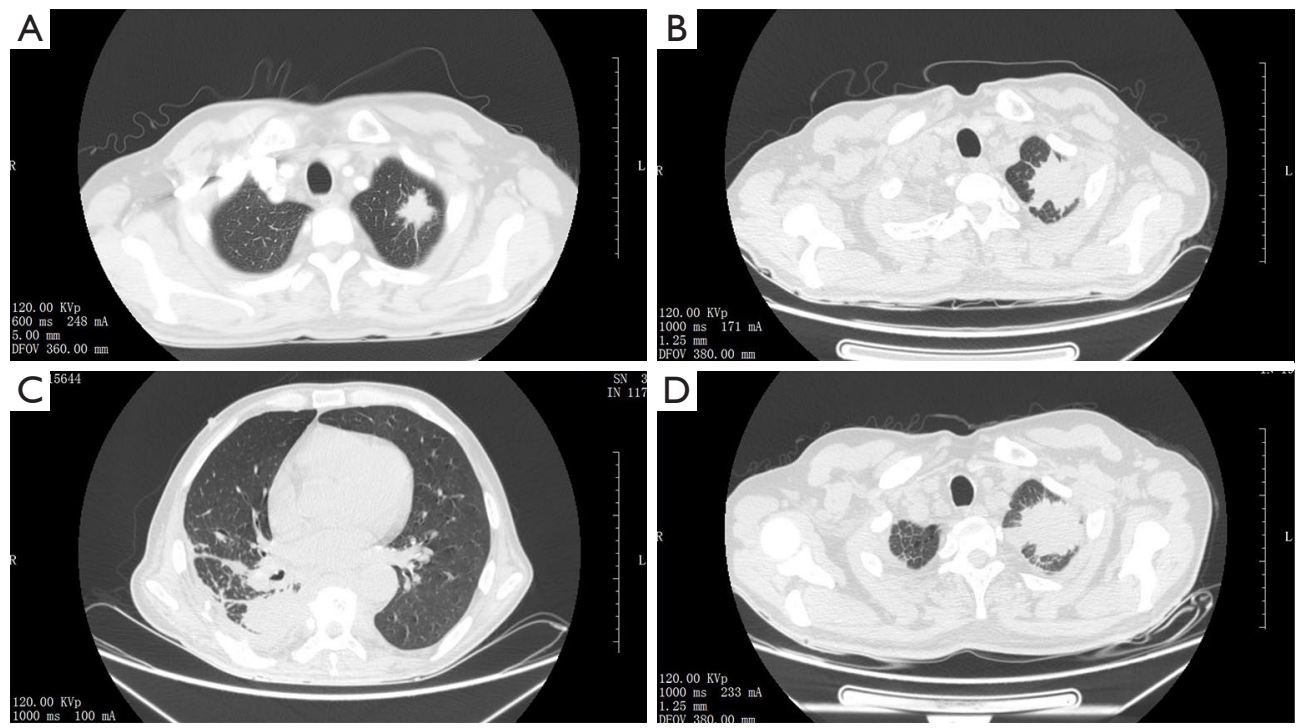

Figure 1 Typical CT images at different time points. (A) The postoperative CT scan on April 1st, 2013; (B) the chest CT scan performed on June 5th, 2013, after the patient was admitted to hospital for the 2 nd time; (C) re-examination of the chest CT on June 27 th after radiotherapy; (D) the chest CT scan performed on September 21st, 2013.

lobe of the right lung was located tightly between the vertebral bodies and adhesions. Thus, it was difficult to perform blunt separation. During the sharp dissection, there was significant bleeding, which was difficult to stop. Based on the view that any further stripping would endanger the patient's life, we decided to stop the resection and close the chest cavity. After the surgery, it was highly suspected that the patient had a lung inflammatory pseudotumor. $\mathrm{He}$ underwent routine postoperative recovery and was discharged after his incision had healed.

On June $4^{\text {th }}$ 2013, the patient was admitted to our hospital for the second time. A chest CT scan performed on June $5^{\text {th }}$ (see Figure $1 B$ ) showed that the left upper lobe nodules were enlarged compared to those in a previous scan of April $1^{\text {st }}, 2013$. Additionally, on the right side, patched high-density shadows were observed in the upper and lower lobes. At this point, the patient was diagnosed with a lung inflammatory pseudotumor. The disease was benign; however, the lesions involved the lungs, thoracic vertebrae, and left humerus, and there was obvious bone destruction. Given the malignant tendency of this pseudotumor and the fact that the patient was experiencing severe pain in his left humerus, the patient underwent radiation therapy, which commenced on June $13^{\text {th }}$, 2013. A total dose of $20 \mathrm{~Gy} / 10 \mathrm{~F}$ was administered.

A re-examination of the patient's chest CT scans on
June $27^{\text {th }}$ after radiotherapy, showed that his left upper lobe nodule and right lung lamella were smaller, and the shadow was lighter (see Figure 1C). Given that an inflammatory pseudotumor is similar to autoimmune diseases, prednisone acetate tablets were administered orally $(30 \mathrm{mg} /$ day for 6 weeks) from June $24^{\text {th }}$ (and then gradually reduced). On July $24^{\text {th }}, 2013$, the patient received a percutaneous puncture biopsy, and an argon-helium knife ablation. Under the guidance of CT scans, the puncture needle removed 1 piece of tissue, and 7 knives were then inserted into the right chest. 2 cycles of treatment $(10 \mathrm{~min} /$ cycle $)$ were administered smoothly (without local edema and bleeding).

The postoperative pathological results showed the chronic inflammation of fibrous tissues, and the formation of inflammatory granulation tissue. From October $14^{\text {th }}$, the patient took compound cyclophosphamide tablets $(50 \mathrm{mg} /$ day) orally for 2 weeks; however, these were discontinued due to obvious bone marrow suppression (the patient had a hemoglobin $(\mathrm{Hb})$ level of $91 \mathrm{~g} / \mathrm{L}$ on October $1^{\text {st }}$, and $71 \mathrm{~g} / \mathrm{L}$ on September $\left.2^{\text {nd }}\right)$. The patient was discharged when the symptom of shortness of breath improved. At this point, there was no palpable swelling of the superficial lymph nodes throughout the patient's body, and no gong sounds were heard in either lung.

On September $21^{\text {st }}, 2013$, the patient was admitted to hospital for further treatment. The diagnosis was as 
follows: (I) a postoperative inflammatory pseudotumor in the thoracic spine and left humerus; (II) lung infection; and (II) a perianal abscess. Chest CT imaging (see Figure 1D) showed the following: (I) the tumor volume in the posterior segment of the left upper lobe was larger than before; (II) the tumor volume in the right lower lobe was larger than before; and (III) the area of multiple patched high-density shadows in both lungs was smaller than before, which suggested that the interstitial lesions had been absorbed, and the bilateral pleural effusion reduced. Additionally, a $3-\mathrm{cm}$ mass (with a soft texture) was observed at 3 o'clock in the anus.

The patient complained that the perianal mass was growing, and significantly affecting his defecation. In relation to the perianal abscess, on September $27^{\text {th }}$, 2013, the patient was placed under sacral anesthesia, and the abscess was incised and drained. The postoperative pathology showed acute purulent inflammation with necrosis in the perianal skin tissue, and the formation of granulation tissues. After the operation, the patient's bowel function recovered, with normal exhaust and defecation, and the wound granulation became fresh. Consequently, the patient was transferred to the Oncology Department for further treatment.

In relation to the patient's lung infection, a sputum culture was taken, and positive Klebsiella pneumoniae results were found. After a drug sensitivity test, the patient received an anti-infective treatment (of Levofloxacin) for 10 days. Routine nutritional support, anemia treatment, and anti-inflammation therapies were provided. After treatment, the results of the sputum culture were negative, and the patient's symptoms of fatigue and shortness of breath improved. The patient was discharged from the hospital on October $11^{\text {th }}, 2013$.

On November $5^{\text {th }}, 2013$, the patient was admitted to our hospital for the 4th time for further treatment of an inflammatory pseudotumor. On this occasion, he was diagnosed with severe anemia. He underwent a red-blood cell transfusion, and was provided with nutritional support and symptomatic treatment. However, after 2 months of blood transfusions, his anemia had not improved significantly. Following a hematological consultation, a recombinant human granulocyte colony stimulating factor (rhGCSF) was used to promote bone marrow erythroid hematopoiesis. Simultaneously, anti-infection and phlegm treatments were routinely administered to treat a pulmonary infection that appeared during hospitalization. After 58 days of treatment, the patient's anemia improved, and he was discharged on January $2^{\text {nd }}, 2014$.
On March $16^{\text {th }}, 2015$, approximately 2 years after the removal of the left humeral inflammatory pseudotumor lesion, the patient experienced aggravated left humerus pain, and was admitted to the hospital for the 5 th time. Magnetic resonance imaging (MRI) of the middle section of the patient's left upper arm on admission indicated a high possibility of chronic osteomyelitis, and a peripheral inflammatory pseudotumor in the left upper arm accompanied by infection. A routine antibiotic treatment was administered as before; however, the effect was poor. Thus, on April $2^{\text {nd }}$, the patient was placed under general anesthesia, and a debridement of the patient's left upperarm osteomyelitis and soft tissue infection was performed. A pathological diagnosis was made. Notably, several pieces of broken gray soft tissue were acquired $(2 \mathrm{~cm} \times 1 \mathrm{~cm} \times$ $0.5 \mathrm{~cm})$. These inflammatory granulation tissues displayed granuloma and purulent necrosis. After the operation, the patient was given supportive treatments to regulate immunity, relieve pain, maintain water, and modulate his electrolyte balance. During his hospitalization, the patient presented with a lung infection and a urinary tract infection. Based on the results of a sputum culture, a urine culture and antimicrobial susceptibility tests, antibiotic therapy was conducted. After more than 2 months of treatment, the patient's condition improved, and he was discharged on May $26^{\text {th }}$.

On June $10^{\text {th }}$, due to breathing difficulties and unconsciousness, the patient was admitted for the $6^{\text {th }}$ time and sent to the Orthopedics Department of our hospital. The final diagnosis was as follows: (I) severe pneumonia and septic shock, with multiple organ dysfunction syndromes, metabolic acidosis, and type II respiratory failure; (II) osteomyelitis of the left humerus with soft tissue infection; (III) urinary system infection; (IV) hypoalbuminemia; (V) incomplete paralysis after surgery of the thoracic spine inflammatory pseudotumor; and (VI) a postoperative right pulmonary pseudotumor. After admission, the patient experienced increasing chest tightness at 16:10. He was sent to the intensive care unit for further treatment. On June $11^{\text {th }}$, under the active treatments of anti-infection, antiinflammatory, expectorant, and an electrolyte imbalance, the patient's condition did not improve significantly. With the consent of his family, active treatment was ceased, and the patient died clinically at 16:36 on June $11^{\text {th }}, 2015$. No autopsy was performed. The main cause of death was regarded as septic shock.

Additionally, this study was performed in conformance to the provisions of the Declaration of Helsinki (as revised 
Table 1 Blood indices at several key time points

\begin{tabular}{lcccc}
\hline Timepoint & $\begin{array}{c}\text { CRP } \\
(\mathrm{mg} / \mathrm{L})\end{array}$ & $\begin{array}{c}\text { Hemoglobin } \\
(\mathrm{g} / \mathrm{L})\end{array}$ & $\begin{array}{c}\text { RBC } \\
\left(10^{9}\right)\end{array}$ & $\begin{array}{c}\text { WBC } \\
\left(10^{9}\right)\end{array}$ \\
\hline $2013-01-16$ & 148.4 & 101 & 3.72 & 9.7 \\
$2013-02-06$ & 97.0 & 83 & 3.12 & 7.5 \\
$2013-02-20$ & 90.6 & 92 & 3.51 & 10.6 \\
$2013-03-17$ & 149.5 & 83 & 3.24 & 10.9 \\
$2013-03-23$ & 95.1 & 88 & 3.41 & 11.5 \\
$2013-04-11$ & 89.9 & 83 & 3.14 & 12.2 \\
$2013-06-05$ & 113.4 & 69 & 3.08 & 16.2 \\
$2013-12-28$ & 7.9 & 106 & 3.48 & 6.4 \\
$2015-03-27$ & 61.6 & 90 & 3.6 & 4 \\
$2015-04-02$ & 50.9 & 75 & 3.06 & 3 \\
$2015-04-27$ & 97.9 & 75 & 2.85 & 4.3 \\
$2015-05-22$ & 51.9 & 83 & 3.01 & 5.9 \\
$2015-06-10$ & 96.4 & 103 & 4 & 17.1 \\
\hline
\end{tabular}

CRP, C-reactive protein; RBC, red blood cells; WBC, white blood cells.

Table 2 Tumor marker levels at several key time points

\begin{tabular}{lccccc}
\hline Timepoint & CEA & AFP & CA199 & Total PSA & Free PSA \\
\hline 2013-01-16 & 1.59 & 2.38 & 8.1 & 0.91 & 0.14 \\
$2013-06-05$ & 1.19 & 2.06 & 7.6 & 0.38 & 0.07 \\
$2013-09-22$ & 1.60 & 2.55 & 11.6 & 0.36 & 0.04 \\
$2013-11-06$ & 1.44 & 2.69 & 4.9 & 0.25 & 0.05 \\
$2015-04-04$ & 2.18 & 1.22 & 51.9 & 0.33 & 0.09
\end{tabular}

CEA, carcinoembryonic antigen; AFP, alpha fetoprotein, CA199, carbohydrate antigen 199; PSA, prostate specific antigen.

in 2013). Written informed consent was obtained from the patient for publication of this study and any accompanying images. All procedures performed in studies involving human participants were in accordance with the ethical standards of the institutional and/or national research committee(s). Tables 1 and 2 show the patient's key blood indices at each time point. Figure 1 shows typical CT images at different time points.

\section{Discussion}

An inflammatory pseudotumor is considered a benign form of lesion marked by a proliferation of myofibroblasts with different degrees of inflammatory infiltrates. It mainly affects the soft tissue and visceral regions of children and young adults. It was first identified in a lung specimen in 1939 , and has been classified as a soft tissue tumor since 2002 (2). Its histopathological features usually include a spectrum of fibroblastic or myofibroblastic proliferation with different infiltrations of inflammatory cells, including plasma cells, lymphocytes, and histiocytes. Generally, it is a rare but benign disease. Indeed, the malignant transformation of PIP has been rarely been reported (2).

The exact etiology of PIP is still unknown. Notably, the direct cause of the present case is not yet fully understood. Generally, PIP may have a number of different causes. It is related to chronic inflammation; acquired immunodeficiency syndrome (AIDS), thymoma surgery, histoplasmosis, syphilis, and interferon treatment (5). However, it is exceedingly rare to have multiple lesions outside the lung (especially over the body). In addition to case reports on chronic inflammation, there have been case reports on iatrogenic inflammatory pseudotumors (e.g., one case of PIP was caused by a medical suture originating from a cardiac surgery that occurred 35 years ago) (6). During his 5 hospital stays, the patient in the case report was observed to have multiple lesions. Previously, the patient had undergone a wedge resection of the right lower lobe after the results of a biopsy suggested a right lower pulmonary pseudotumor. This occurred in May 2011, which suggests that the disease had been developing for more than 4 years. Exposure to chronic inflammation may have played an essential role in PIP formation in this case.

PIPs can be challenging to diagnose. In the present case, an inflammatory pseudotumor was consistently diagnosed based on the pathological results of tissues from the right lower lobe (resected in May 2011), the thoracic spine, the left humerus, and the left upper lobe. The data confirmed that the patient had a rare PIP case with a high malignancy.

PIPs are generally treated surgically, and the prognosis of patients undergoing complete surgical resection is favorable. However, this patient underwent multiple surgical operations on multiple lesions (distributed in the right lung, left lung, thoracic spine, and left humerus). We are of the view that the patient's final poor prognosis was related to the lack of effective systemic treatment. For example, glucocorticoid therapy can help relieve symptoms of inflammatory pseudotumor according to published literature reports, and in some cases, the lesions may disappear (7-9). However, in this case, hormone therapy 
only slightly relieved the symptoms, and lesion size and anemia did not significantly improve. Additionally, the patient discontinued usage of cyclophosphamide due to bone marrow suppression. To date, cyclophosphamide has not been successfully used to treat PIPs. However, patients with systemic multiple lesions may tolerate and benefit from this therapy of they have strong bone marrow hematopoietic function. Finally, given the obvious malignant tendency, local radiotherapy can also be attempted in addition to surgery (10). In the present case, radiotherapy relieved some symptoms, but it could not control the progression of PIP. Unfortunately, the patient died in the short- to middleterm. Future studies should seek to examine the longterm effects of PIPs. Local cryoablation could be tried on patients who cannot tolerate surgery.

Someone could argue the necessity of multiple operations. Although it was reported that PIP can be controlled by surgery and drugs, most of the studies are based on the condition of only one lesion. To date, the routine treatment of inflammatory pseudotumor is majorly based on surgery. In this case, the patient had multiple lesions; he had been treated with drugs based on known information, but symptoms only improved slightly, no significant change in imaging assessment was observed. Therefore, we used surgery when necessary. After the operations, pain was relieved in back and left humerus and the quality of life was improved. The condition was controlled temporarily.

\section{Conclusions}

This case report presented a rare case of PIP in a middleage male patient with significant progression to a malignant form. Further research should be conducted on this form of PIP. Multiple-lesion PIP does not respond to systemic treatment; thus, PIP can be highly fatal. Early and aggressive treatment is necessary, and multidisciplinaryteam treatments are recommended.

\section{Acknowledgments}

Funding: None.

\section{Footnote}

Reporting Checklist: The authors have completed the CARE reporting checklist. Available at http://dx.doi.org/10.21037/ tcr-21-564
Conflicts of Interest: All authors have completed the ICMJE uniform disclosure form (available at http://dx.doi. org/10.21037/tcr-21-564). The authors have no conflicts of interest to declare.

Ethical Statement: The authors are accountable for all aspects of the work in ensuring that questions related to the accuracy or integrity of any part of the work are appropriately investigated and resolved. Written informed consent was obtained from the patient for publication of this study and any accompanying images. All procedures performed in studies involving human participants were in accordance with the ethical standards of the institutional and/or national research committee(s) and with the Helsinki Declaration (as revised in 2013).

Open Access Statement: This is an Open Access article distributed in accordance with the Creative Commons Attribution-NonCommercial-NoDerivs 4.0 International License (CC BY-NC-ND 4.0), which permits the noncommercial replication and distribution of the article with the strict proviso that no changes or edits are made and the original work is properly cited (including links to both the formal publication through the relevant DOI and the license). See: https://creativecommons.org/licenses/by-nc-nd/4.0/.

\section{References}

1. Braham Y, Migaou A, Njima M, et al. Inflammatory myofibroblastic tumor of the lung: A rare entity. Respir Med Case Rep 2020;31:101287.

2. Inamdar AA, Pulinthanathu R. Malignant transformation of inflammatory myofibroblastic tumor of urinary bladder: A rare case scenario. Bladder (San Franc) 2019;6:e39.

3. Jain N, Bharat A, Marenych N, et al. Calcifying Fibrous Tumor Complicated by Empyema. Cureus 2020;12:e8729.

4. Rodrigues L, Domingues R, Amaral D, et al. Primary Pulmonary Tumor in an Adolescent. Acta Med Port 2020;33:512-5.

5. Govender D, Jackson C, Chetty D. Syphilitic Pulmonary Inflammatory Pseudotumor: A Diagnostic Challenge. Int J Surg Pathol 2021;29:90-6.

6. Meng S, Liu G, Wang S, et al. Case report: inflammatory pseudotumor in the lung parenchyma caused by a medical suture originating from a cardiac surgery 35 years ago. J Cardiothorac Surg 2020;15:151.

7. Bhattad PB, Joseph DL, Peterson E. IgG4-Related Disease Manifesting as Hypocomplementemic 
Tubulointerstitial Nephritis: A Rare Case Report and Literature Review. J Investig Med High Impact Case Rep 2020;8:2324709620952213.

8. Liu A, Zhang Q, Liu B, et al. A case of immunoglobulin G4-related lung disease with bilateral diffuse infiltration: A case report. Medicine (Baltimore) 2017;96:e9211.

9. Na YS, Park SG. Inflammatory myofibroblastic tumor of the pleura with adjacent chest wall invasion and metastasis to the kidney: a case report. J Med Case Rep 2018;12:253.

10. Motooka Y, Fujino K, Gregor A, et al. Endobronchial Ultrasound-Guided Radiofrequency Ablation of Lung Tumors and Mediastinal Lymph Nodes: A Preclinical Study in Animal Lung Tumor and Mediastinal Adenopathy Models. Semin Thorac Cardiovasc Surg 2020;32:570-8.

(English Language Editor: L. Huleatt)
Cite this article as: Li N, Zou C, Gao S, Guo Y, Wang W, Guo Y, Zhang J, Wang C, An Y. A fatal multiple-lesion, inflammatory, pseudotumor in the lung: a rare case report. Transl Cancer Res 2021;10(4):1947-1953. doi: 10.21037/tcr-21-564 